\title{
MENISCAL REPAIR
}

\section{AN ARTHROSCOPIC TECHNIQUE}

\author{
F. ALAN BARBER, ROBERT G. STONE
}

\section{From The University of Texas Health Science Center at Dallas}

\begin{abstract}
Repair of a longitudinal peripheral meniscal tear permits salvage of this structure in a high percentage of cases. In previous reports an arthrotomy was used to accomplish this repair; in this paper an arthroscopic technique is presented. The potential risks are significant and include damage to the peroneal nerve and popliteal vascular structures, failure of meniscal healing and the usual complications of arthroscopy. The procedure is recommended only for the advanced arthroscopist who is advised first to establish clearly by cadaver dissections the anatomical relationships.
\end{abstract}

The importance of the meniscus in the knee has become increasingly apparent in recent years as additional knowledge of its multiple functions has been gained. It provides stability, transfers loads, lubricates, and facilitates the nutrition of articular cartilage, to name only a few roles (Allen 1980; McGinty 1980). Recent reports have emphasised the long-term consequences of losing both the medial meniscus and the anterior cruciate ligament (Hughston and Barrett 1983; Lynch, Henning and Glick 1983). Because of these factors, a determined effort to preserve the meniscus is being made whenever possible. Stone (1979), Wirth (1981), Hamberg, Gillquist and Lysholm (1983) and others have reported their experience with open meniscal repairs; the incidence of meniscal healing has been reported to be $75 \%$ for chronic peripheral tears and higher for acute tears.

Many reports also document the healing potential of meniscal tears away from the synovial meniscal junction. Arnoczky and Warren (1982) have demonstrated that in human cadavers the outer $10 \%$ to $20 \%$ of the meniscus has a blood supply (the perimeniscal capillary plexus). Clark and Ogden (1983) recently reported that the vascularity extended to the inner rim in the fetal meniscus. These reports suggest an agedependent lessening of the extent of the blood supply. It seems likely therefore that, in the second and third decades, the meniscus has a more extensive capillary plexus than was seen in the cadaver studies of Arnoczky and Warren, whose subjects were much older. This more extensive perimeniscal plexus should increase the possi-

F. A. Barber, MD, FACS, Clinical Assistant Professor

R. G. Stone, MD, Clinical Associate Professor

Division of Orthopedic Surgery, The University of Texas Health Science Center, Dallas, Texas, USA.

Requests for reprints should be sent to F. A. Barber, MD,

Plano Orthopedic and Sports Medicine Center, 1524 Independence Parkway. Suite C, Plano, Texas 75075, USA.

(C) 1985 British Editorial Society of Bone and Joint Surgery $0301-620 \mathrm{X} / 85 / 1024 \$ 2.00$ bility of healing if the meniscus can be reapproximated by sutures and protected.

The standard operative approach requires a posteromedial or posterolateral arthrotomy for the placement of sutures. This technique makes it especially difficult to approach extensive longitudinal tears of the medial meniscus because of the presence of the medial collateral

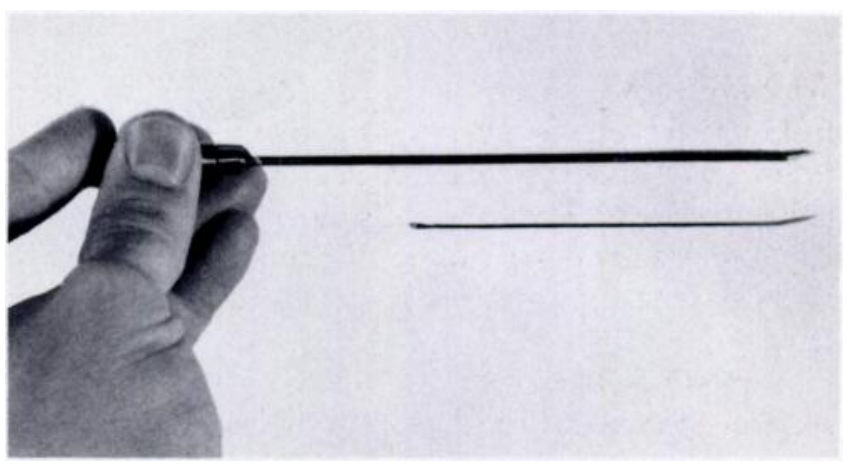

Fig. 1

The Henning needle-holder is shown with a suitably bent Keith needle.

ligament. In order to facilitate repairs of these longitudinal tears, Henning (personal communication) devised an arthroscopic technique using a special needleholder of his own design (Fig. 1). A modification of this arthroscopic technique has been used by us since late 1982 to repair both synovial-meniscal junction disruptions and intrasubstance tears of the meniscus. To date, although the follow-up is short, there have been no retears in this group. Encouraged by these results we report the surgical technique presently used.

\section{TECHNIQUE}

The standard video arthroscopy set-up is used. A sturdy leg-holder and an assistant to stress and position the leg are necessary to permit adequate access to the entire synovial-meniscal junction. In order to be able to see 
active bleeding from the perimeniscal capillary plexus the tourniquet is not inflated.

The longitudinal tear is identified and defined by the probe. The length and distance from the periphery of the tear is noted and reduction of the bucket-handle is accomplished if needed. Once the inner rim portion is properly positioned, the following technique for suturing is used.

A hollow inflow cannula (which permits easy repeated access and prevents tangling the suture in the soft tissues) is inserted through the anterolateral or midpatellar lateral portal, positioned in the medial compartment and aligned towards the meniscal tear. The best access is obtained if the knee is held in $10^{\circ}$ of flexion and a valgus stress is applied.

A $3 \mathrm{~mm}$ basket forceps is inserted through the cannula and used to debride the outer rim of the meniscus until bleeding from the perimeniscal capillary plexus is seen (Fig. 2). A $7.6 \mathrm{~cm}$ Keith needle is bent to a 30 angle $1 \mathrm{~cm}$ from its tip and threaded with a 3-0

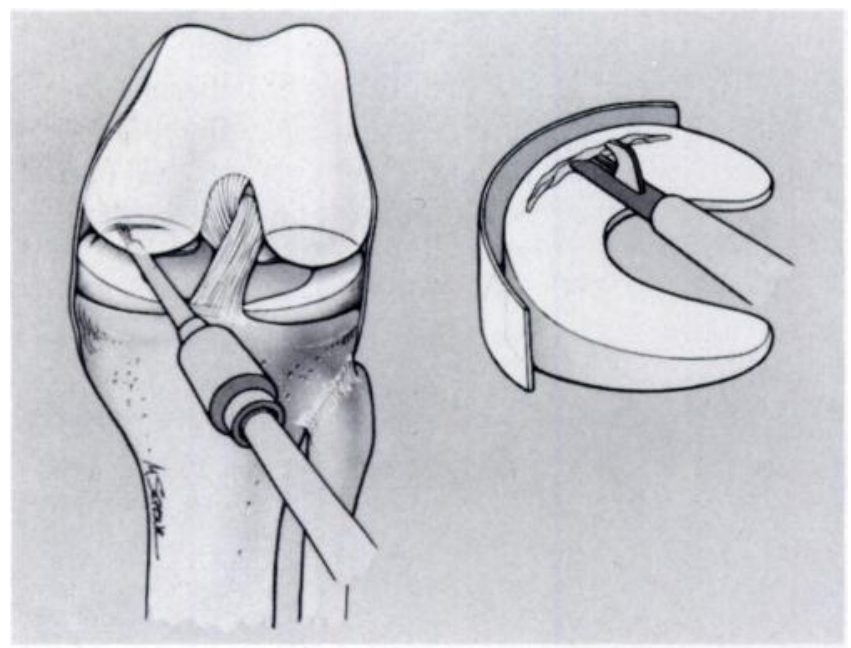

Fig. 2

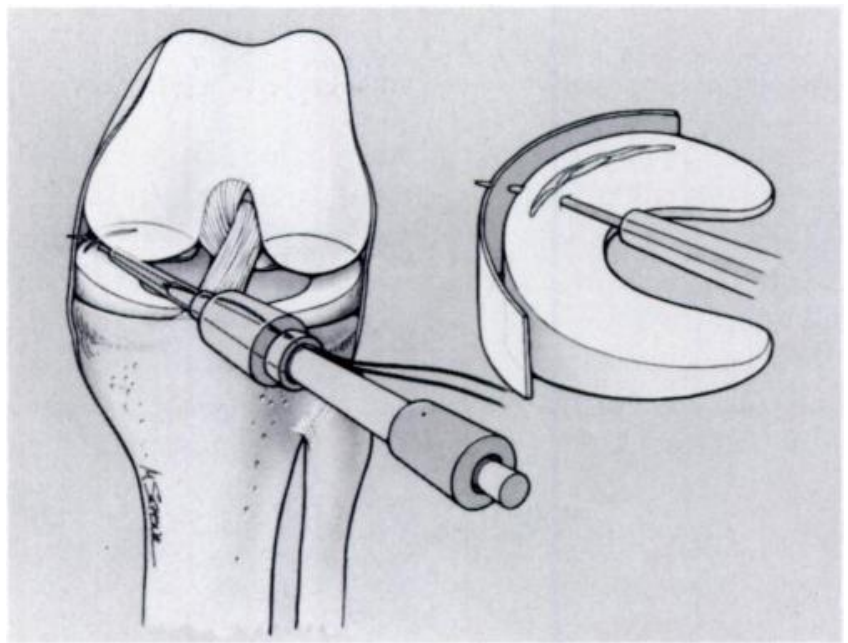

Fig. 3

Figure 2-A basket forceps inserted through a cannula is used to debride the outer rim back to the perimeniscal capillary plexus. Figure 3-The Henning needle-holder is used to pass the threaded Keith needle through the torn meniscus and out through the capsule.
Mersilene suture. The threaded Keith needle is placed in a Henning needle-holder and advanced through the cannula with the curve directed anteriorly. The needle is passed through the superior surface of the meniscal bucket-handle about $2 \mathrm{~mm}$ in from the tear and advanced through the tear and into the medial capsule (Fig. 3). Placement can be facilitated by rotating the Keith needle slightly to take advantage of its distal curve.

Care is taken to ensure that the needle will emerge from the skin anterior to the semitendinosus tendon. The index finger protected by a sewing thimble can be placed behind the tendon to protect the popliteal artery. As the Keith needle is advanced, it tents the skin. At this point a knife is used to make a portal. The needle is pulled through with the needle-holders and the suture unthreaded. An ample length of suture should still remain outside the cannula to permit rethreading of the Keith needle. The standing end of the suture is then threaded, the needle is placed in the Henning needle-holder, and the needle is passed again in the same manner through the bucket-handle, outer rim and capsule, and out through the previously made portal. A Z-retractor placed in the posteromedial portal acts as a neurovascular shield and needle guide, and facilitates the exit of this and subsequent sutures through a single portal. The second pass of this suture should be placed $2 \mathrm{~mm}$ lateral to the first, creating a horizontal mattress-suture (Fig. 4).

A separate suture is needed for every $5 \mathrm{~mm}$ length of tear above $1 \mathrm{~cm}(\mathrm{eg}$, a $20 \mathrm{~mm}$ tear needs 3 sutures, a $25 \mathrm{~mm}$ tear needs 4). Once all the sutures have been placed, they are pulled tight to approximate the edges of the torn meniscus and capsule (Fig. 5). If the repair is judged adequate, the matched sutures are tied snugly through the portal incision. A curved clamp is used to free the skin and subcutaneous tissues and so prevent puckering. The portal is closed in the routine manner and the knee is immobilised in $45^{\circ}$ of flexion for six weeks.

For the lateral meniscus the technique is similar,

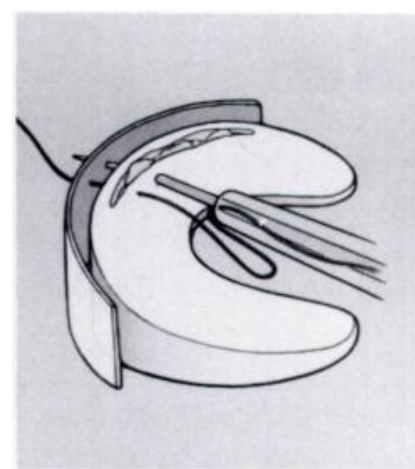

Fig. 4

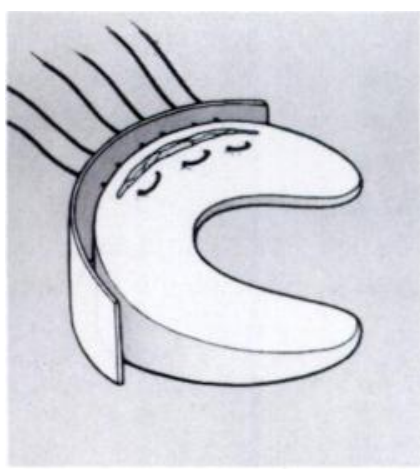

Fig. 5
Figure 4-The proximal end of the suture is then threaded onto the Keith needle and, using the same technique, a horizontal mattresssuture is created. Figure 5-Once all the sutures have been placed, they are pulled tight and tied through the portal incision. 
except that care is taken to ensure that the Keith needle emerges anterior to the biceps tendon so as to avoid injury to the peroneal nerve.

\section{DISCUSSION}

This is a new technique that is still undergoing refinement and until long-term results are available it should be considered developmental. It permits repair and salvage of the torn meniscus by an arthroscopic procedure. With experience, it will make repair easier and enable sutures to be placed more accurately than with the open technique. The disadvantages include entrapment of soft tissues by the tied suture (although in our personal experience with 24 cases this has not been a problem) and the potential for serious complications; these include injury to the peroneal nerve, injury to the popliteal artery, failure to heal and the usual complications of arthroscopy. It is important to emphasise that the Keith needles should emerge anterior to the biceps tendon laterally or the semitendinosus tendon medially in order to avoid these problems. The technique is demanding and suggested only for the advanced arthroscopist. Even the experienced arthroscopic surgeon preparing for this technique is well advised to establish the anatomical relationships in his mind by cadaver dissections. Unless the anatomical relationships are respected, the potential for disastrous complications exists. It cannot be emphasised strongly enough that the errant needle could cause injury to the popliteal artery or peroneal nerve.

Non-absorbable sutures are presently being used because in one of the early cases a Vicryl suture was seen to loosen rapidly when exposed to synovial fluid. A hinged knee-brace fixed at $45^{\circ}$ is used postoperatively for six weeks; after three weeks the brace is adjusted to permit a $30^{\circ}$ to $60^{\circ}$ arc of movement. After removal of the brace, a graduated rehabilitation programme reestablishes muscle power, but vigorous sports are forbidden for six months.

To be suitable for this technique, the tear should be a single longitudinal one extending completely through the substance of the meniscus and situated either at the synovial-meniscal junction or within $3 \mathrm{~mm}$ of it. Tears under $1 \mathrm{~cm}$ in length are treated in a brace without suture. Multiple longitudinal tears and single tears with a damaged inner rim are not considered suitable for repair. Longitudinal tears situated 4 to $5 \mathrm{~mm}$ into the substance of the meniscus are currently being investigated. While no long-term results are available with this technique, reports of open meniscal repairs are very encouraging (Wirth 1981; Hamberg et al. 1983). The arthroscopic technique should provide equivalent results but with shorter periods in hospital and less discomfort.

\section{REFERENCES}

Allen WC. Medial meniscus injuries: mechanism, diagnosis, and treatment. In: AAOS Symposium on the athlete's knee: surgical repair and reconstruction. St Louis: CV Mosby Company, 1980: $131-40$.

Arnoczky SP, Warren RF. Microvasculature of the human meniscus. Am J Sports Med 1982;10:90-5.

Clark CR, Ogden JA. Development of the menisci of the human knee joint: morphological changes and their potential role in childhood meniscal injury. J Bone Joint Surg $[\mathrm{Am}]$ 1983;65-A:538-47.

Hamberg P, Gillquist J, Lysholm J. Suture of new and old peripheral meniscus tears. J Bone Joint Surg $[$ Am] 1983;65-A:193-7.
Hughston JC, Barrett GR. Acute anteromedial rotatory instability: long-term results of surgical repair. J Bone Joint Surg [Am] $1983 ; 65-A: 145-53$.

Lynch MA, Henning CE, Glick KR Jr. Knee joint surface changes: long-term follow-up meniscus tear treatment in stable anterior cruciate ligament reconstructions. Clin Orthop 1983;172:142-53.

McGinty JB. Partial meniscectomy. In: AAOS Symposium on the athlete's knee: surgical repair and reconstruction. St Louis: CV Mosby Company, 1980:112-4.

Stone RG. Peripheral detachment in the menisci of the knee: a preliminary report. Orthop Clin North Am 1979;10(3):643-57.

Wirth CR. Meniscus repair. Clin Orthop 1981;157:153-60. 